\title{
Duality based boundary conditions and dual consistent finite difference discretizations of the Navier-Stokes and Euler equations
}

Jens Berg and Jan Nordström

\section{Linköping University Post Print}

\section{Tweet}

N.B.: When citing this work, cite the original article.

Original Publication:

Jens Berg and Jan Nordström, Duality based boundary conditions and dual consistent finite difference discretizations of the Navier-Stokes and Euler equations, 2013, Journal of Computational Physics.

http://dx.doi.org/10.1016/j.jcp.2013.11.031

Copyright: Elsevier

http://www.elsevier.com/

Postprint available at: Linköping University Electronic Press

http://urn.kb.se/resolve?urn=urn:nbn:se:liu:diva-102292 


\title{
Duality based boundary conditions and dual consistent finite difference discretizations of the Navier-Stokes and Euler equations
}

\author{
Jens Berg* \\ Uppsala University, Department of Information Technology, SE-751 05, Uppsala, Sweden \\ Jan Nordström \\ Linköping University, Department of Mathematics, SE-581 83, Linköping, Sweden
}

\begin{abstract}
In this paper we derive new farfield boundary conditions for the time-dependent Navier-Stokes and Euler equations in two space dimensions. The new boundary conditions are derived by simultaneously considering well-posedess of both the primal and dual problems. We moreover require that the boundary conditions for the primal and dual Navier-Stokes equations converge to well-posed boundary conditions for the primal and dual Euler equations.

We perform computations with a high-order finite difference scheme on summationby-parts form with the new boundary conditions imposed weakly by the simultaneous approximation term. We prove that the scheme is both energy stable and dual consistent and show numerically that both linear and non-linear integral functionals become superconvergent.
\end{abstract}

Keywords: High order finite differences; Summation-by-parts; Superconvergence; Boundary conditions; Dual consistency; Stability

${ }^{*}$ Corresponding author: Jens Berg

Address: Division of Scientific Computing, Department of Information Technology, Uppsala University, Box 337, SE-751 05, Uppsala, Sweden

Phone: +46 $18-4716253$

Fax: +46 $18523049,+4618511925$

E-mail: jens.berg@it.uu.se

Preprint submitted to Elsevier

November 24, 2013 


\section{Introduction}

The focus on this paper is the derivation of new boundary conditions for the Navier-Stokes and Euler equations. The technique that will be used is, however, general and can be applied to any initial boundary value problem (IBVP). In particular, we focus on deriving farfield boundary conditions for the Navier-Stokes equations which converge to well-posed boundary conditions the Euler equations in the limit of vanishing viscosity for the difficult subsonic flow case.

There are many numerical methods that can be used to obtain approximate solutions to IBVPs. In this paper, the focus will be on the finite difference method on summation-by-parts (SBP) form with weak implementation of the boundary conditions. The finite difference method is appealing due to its high-order accuracy and ease of implementation.

The finite difference method on SBP form were originally developed by Kreiss and Scherer $[19,20]$ as a means to mimic integration by parts, and to construct high-order accurate energy stable finite difference schemes for linearly well-posed hyperbolic problems. The implementation of the boundary conditions were made feasible by Carpenter et al. in [5] by adding the boundary conditions as penalty terms, the so called simultaneous approximation terms (SATs). The combination of SBP and SAT allows for energy stable finite difference discretizations of any linearly well-posed IBVP which is independent of the order of accuracy. Details on the construction and properties of the SBP operators can be found in [39] for the first derivative and in $[24,22]$ for the second derivative.

The SBP-SAT technique has been extended to include curvilinear coordinate transforms [32, 42], multi-block couplings [6, 31, 7, 34, 23, 28], artificial dissipation operators $[26,8]$, and has been applied to numerous applications where it has proven to be robust. See for example $[44,25,14,16]$.

The most recent development of the SBP-SAT technique were made by Hicken and Zingg $[13,12]$. They analyzed the properties of the discrete norm and showed that superconvergent linear volume integral functionals of linear IBVPs could be computed from so called dual consistent SBP-SAT discretizations. In general, the solution to an IBVP with a diagonal norm is accurate of order $p+1$ since the interior accuracy is $2 p$ with $p$ th-order boundary accuracy [45]. It was shown in [12], and later extended in [3], that linear integral functionals from a diagonal norm dual consistent SBP-SAT discretization retains the full accuracy of $2 p$. Dual consistency is a matter of choosing the coefficients in the SATs and does not increase the computational complexity. Superconvergence of linear integral functionals hence comes for free. This theory is still new and work remains to be done for non-linear IBVPs, nonlinear functionals, boundary integrals such as lift and drag, and higher-dimensional 
problems.

Free superconvergence is an attractive property of a dual consistent SBP-SAT discretization. The duality concept can, however, also be used to construct new boundary conditions for the continuous problem. By having advanced boundary conditions for the continuous problem, the numerical scheme can be greatly enhanced $[29,30,4]$.

The superconvergence property have only been proven for linear problems with linear integral functionals. In this paper we will use the linearized Navier-Stokes and Euler equations to derive schemes which will be applied to the non-linear problems and non-linear integral functionals. The linear theory will hence be applied to nonlinear problems for which the theory is still incomplete. It was shown in [3] that already for a coarse mesh, the accuracy of linear integral functionals of dual consistent schemes were superior to the dual inconsistent case, even though the solutions were of the same accuracy.

The aim of this paper is threefold. The first goal is to use duality to construct advanced far-field boundary conditions for the Navier-Stokes and Euler equations in two space dimensions. The second is to use the new boundary conditions in an SBP-SAT discretization and show that dual consistency and energy stability becomes equivalent The third is to show computationally that integral functionals are superconvergent even when applied to non-linear problems.

\section{The Navier-Stokes equations}

The two-dimensional time-dependent compressible Navier-Stokes equations in non-dimensional form can be written as

$$
q_{t}+F_{x}^{I}+G_{y}^{I}=\varepsilon\left(F_{x}^{V}+G_{y}^{V}\right)
$$

where $\varepsilon=M a / R e$ is the ratio between the Mach and Reynolds number, $q=$ $[\rho, \rho u, \rho v, e]^{T}$ are the conservative variables and $\rho, u, v, e$ are the density, velocities, and energy, respectively. The energy is defined as

$$
e=\frac{p}{\gamma-1}+\frac{1}{2} \rho\left(u^{2}+v^{2}\right)
$$

where $\gamma$ is the ratio of specific heats and $p$ is the pressure. We assume an ideal fluid and hence the equation of state is

$$
\gamma p=\rho T
$$


where $T$ is the temperature. The above variables have been non-dimensionalized using

$$
\rho=\frac{\rho^{*}}{\rho_{\infty}^{*}}, \quad u=\frac{u^{*}}{c_{\infty}^{*}}, \quad v=\frac{v^{*}}{c_{\infty}^{*}}, \quad e=\frac{e^{*}}{\rho_{\infty}^{*}\left(c_{\infty}^{*}\right)^{2}}, \quad p=\frac{p^{*}}{\rho_{\infty}^{*}\left(c_{\infty}^{*}\right)^{2}}, \quad T=\frac{T^{*}}{T_{\infty}^{*}},
$$

where the $*$-superscript denotes a dimensional variable and the $\infty$-subscript the free stream reference value. The inviscid fluxes are given by

$$
F^{I}=\left[\begin{array}{c}
\rho u \\
p+\rho u^{2} \\
\rho u v \\
(p+e) u
\end{array}\right], \quad G^{I}=\left[\begin{array}{c}
\rho v \\
\rho u v \\
p+\rho v^{2} \\
(p+e) v
\end{array}\right]
$$

and the viscous fluxes are given by

$$
F^{V}=\left[\begin{array}{c}
0 \\
\tau_{x x} \\
\tau_{x y} \\
u \tau_{x x}+v \tau_{x y}+\frac{\kappa}{\operatorname{Pr}(\gamma-1)} T_{x}
\end{array}\right], \quad G^{V}=\left[\begin{array}{c}
0 \\
\tau_{y x} \\
\tau_{y y} \\
u \tau_{y x}+v \tau_{y y}+\frac{\kappa}{\operatorname{Pr}(\gamma-1)} T_{y}
\end{array}\right] \text {, }
$$

where $\kappa$ is the thermal conduction coefficient, and $\operatorname{Pr}$ the Prandtl number. The stress tensor is

$$
\begin{aligned}
\tau_{x x} & =2 \mu \frac{\partial u}{\partial x}+\lambda\left(\frac{\partial u}{\partial x}+\frac{\partial v}{\partial y}\right), \quad \tau_{x y}=\tau_{y x}=\mu\left(\frac{\partial u}{\partial y}+\frac{\partial v}{\partial x}\right), \\
\tau_{y y} & =2 \mu \frac{\partial v}{\partial y}+\lambda\left(\frac{\partial u}{\partial x}+\frac{\partial v}{\partial y}\right),
\end{aligned}
$$

where $\mu, \lambda$ are the dynamic and second viscosity, respectively. Since (1) is a highly non-linear system of equations, it can not be easily analyzed using the energy method. Instead, the analysis will be performed on the linearized, symmetrized, and frozen coefficient system. We begin by considering the non-conservative system in primitive variables $Q=[\rho, u, v, p]^{T}$ and apply the parabolic symmetrizer in [1]. A linearization $Q=\bar{U}+U^{\prime}$ is introduced where $\bar{U}=[\bar{\rho}, \bar{u}, \bar{v}, \bar{p}]^{T}$ denotes a constant state and $U^{\prime}=$ $\left[\rho^{\prime}, u^{\prime}, v^{\prime}, p^{\prime}\right]$ a perturbation around $\bar{U}$. The result is the linear, constant coefficient, and symmetric system

$$
U_{t}^{\prime}+A U_{x}^{\prime}+B U_{y}^{\prime}=\varepsilon\left(\left(C_{11} U_{x}^{\prime}+C_{12} U_{y}^{\prime}\right)_{x}+\left(C_{21} U_{x}^{\prime}+C_{22} U_{y}^{\prime}\right)_{y}\right),
$$


where

$$
\begin{aligned}
& U^{\prime}=\left[\frac{\bar{c}}{\bar{\rho} \sqrt{\gamma}} \rho^{\prime}, u^{\prime}, v^{\prime}, \frac{1}{\bar{c} \sqrt{\gamma(\gamma-1)}} T^{\prime}\right]^{T} \\
& A=\left[\begin{array}{cccc}
\bar{u} & \frac{\bar{c}}{\sqrt{\gamma}} & 0 & 0 \\
\bar{c} & \bar{u} & 0 & \bar{c} \sqrt{\frac{\gamma-1}{\gamma}} \\
0 & 0 & \bar{u} & 0 \\
0 & \bar{c} \sqrt{\frac{\gamma-1}{\gamma}} & 0 & \bar{u}
\end{array}\right], \quad C_{11}=\left[\begin{array}{cccc}
0 & 0 & 0 & 0 \\
0 & \frac{\lambda+2 \mu}{\bar{\rho}} & 0 & 0 \\
0 & 0 & \frac{\mu}{\bar{\rho}} & 0 \\
0 & 0 & 0 & \frac{\gamma \mu}{\operatorname{Pr} \bar{\rho}}
\end{array}\right], \\
& B=\left[\begin{array}{cccc}
\bar{v} & 0 & \frac{\bar{c}}{\sqrt{\gamma}} & 0 \\
0 & \bar{v} & 0 & 0 \\
\frac{\bar{c}}{\sqrt{\gamma}} & 0 & \bar{v} & \bar{c} \sqrt{\frac{\gamma-1}{\gamma}} \\
0 & 0 & \bar{c} \sqrt{\frac{\gamma-1}{\gamma}} & \bar{v}
\end{array}\right], \quad C_{22}=\left[\begin{array}{cccc}
0 & 0 & 0 & 0 \\
0 & \frac{\mu}{\bar{\rho}} & 0 & 0 \\
0 & 0 & \frac{\lambda+2 \mu}{\bar{\rho}} & 0 \\
0 & 0 & 0 & \frac{\gamma \mu}{\operatorname{Pr} \bar{\rho}}
\end{array}\right] \text {, }
\end{aligned}
$$

and

$$
C_{12}=C_{21}=\left[\begin{array}{cccc}
0 & 0 & 0 & 0 \\
0 & 0 & \frac{\lambda+\mu}{2 \bar{\rho}} & 0 \\
0 & \frac{\lambda+\mu}{2 \bar{\rho}} & 0 & 0 \\
0 & 0 & 0 & 0
\end{array}\right]
$$

Linearizing the equation of state (2) gives the linearized equation of state

$$
\gamma p^{\prime}=\bar{T} \rho^{\prime}+\bar{\rho} T^{\prime}
$$

To ease the notation, all primed superscripts will be dropped and we consider only the perturbed variables.

To avoid additional difficulties in deriving the new boundary conditions due to geometric considerations, we let the domain of interest be the unit square. We assume that the flow field has been linearized around a state with $\bar{u}, \bar{v} \geq 0$, see Figure 1 . Furthermore, we are interested in the subsonic case where $\bar{u}, \bar{v}<\bar{c}$. We will consider boundary conditions of far-field type,

$$
\begin{aligned}
H_{W} U-\varepsilon\left(C_{11} U_{x}+C_{12} U_{y}\right)=0, & H_{E} U+\varepsilon\left(C_{11} U_{x}+C_{12} U_{y}\right)=0, \\
H_{S} U-\varepsilon\left(C_{21} U_{x}+C_{22} U_{y}\right)=0, & H_{N} U+\varepsilon\left(C_{21} U_{x}+C_{22} U_{y}\right)=0,
\end{aligned}
$$

where the matrices $H_{W, E, S, N}$ are to be constructed such that the primal and dual problems for both the Navier-Stokes and Euler equations, as $\varepsilon \rightarrow 0$, are well-posed. The subscripts W, E, S, N refer to the west, east, south, and north boundaries, respectively. 


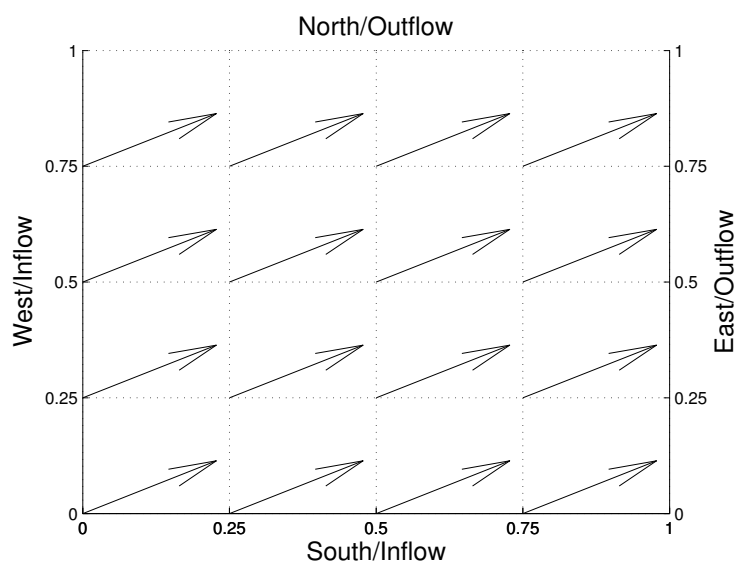

Figure 1: Computational domain and flow assumptions

\section{The dual Navier-Stokes equations}

To derive the dual Navier-Stokes equations we consider (3) in the form

$$
\begin{aligned}
U_{t}+L U & =F, & & (x, y) \in \Omega, \\
B U & =0, & & (x, y) \in \Gamma \subseteq \partial \Omega, \\
U & =0, & t & =0, \\
J(U) & =(G, U) . & &
\end{aligned}
$$

In (6), $J(U)$ is a linear integral functional with a weight function $G$ and $B$ implements the boundary conditions in (5). The right-hand side $F$ may be identically zero, but a symbol is needed to perform integration by parts when deriving the dual problem. The differential operator $L$ is given by

$$
L=A \frac{\partial}{\partial x}+B \frac{\partial}{\partial y}-\varepsilon\left(\left(C_{11} \frac{\partial}{\partial x}+C_{12} \frac{\partial}{\partial y}\right) \frac{\partial}{\partial x}+\left(C_{21} \frac{\partial}{\partial x}+C_{22} \frac{\partial}{\partial y}\right) \frac{\partial}{\partial y}\right) .
$$


We seek a function $\Theta=\left[\theta_{1}, \theta_{2}, \theta_{3}, \theta_{4}\right]^{T}$ such that $\int_{0}^{T} J(U) d t=\int_{0}^{T}(\Theta, F)$, and we get by the Gauss-Green formula

$$
\begin{aligned}
& \int_{0}^{T} J(U) d t=\int_{0}^{T}(G, U)-\int_{0}^{T}\left(\Theta, U_{t}+L U-F\right) d t \\
& =\int_{0}^{T}(\Theta, F) d t+\int_{0}^{T}\left(\Theta_{t}-L^{*} \Theta+G, U\right) d t \\
& +\int_{0}^{T} \int_{W} \Theta^{T}\left(A U-\varepsilon\left(C_{11} U_{x}+C_{12} U_{y}\right) d y d t+\varepsilon \int_{0}^{T} \int_{W}\left(\Theta_{x}^{T} C_{11}+\Theta_{y}^{T} C_{12}\right) U d y d t\right. \\
& -\int_{0}^{T} \int_{E} \Theta^{T}\left(A U-\varepsilon\left(C_{11} U_{x}+C_{12} U_{y}\right) d y d t-\varepsilon \int_{0} \int_{E}\left(\Theta_{x}^{T} C_{11}+\Theta_{y}^{T} C_{12}\right) U d y d t\right. \\
& +\int_{0}^{T} \int_{S} \Theta^{T}\left(B U-\varepsilon\left(C_{21} U_{x}+C_{22} U_{y}\right) d x d t+\varepsilon \int_{0} \int_{S}\left(\Theta_{x}^{T} C_{21}+\Theta_{y}^{T} C_{22}\right) U d x d t\right. \\
& -\int_{0}^{T} \int_{N}^{T} \Theta^{T}\left(B U-\varepsilon\left(C_{21} U_{x}+C_{22} U_{y}\right) d x d t-\varepsilon \int_{0} \int_{N}\left(\Theta_{x}^{T} C_{21}+\Theta_{y}^{T} C_{22}\right) U d x d t\right. \\
& -\int_{\Omega}\left[\Theta^{T} U\right]_{t=T} d \Omega,
\end{aligned}
$$

where $\int_{W, E, S, N}$ denotes integration over the west, east, south, and north boundary, respectively. The dual operator, $L^{*}$, is given by

$$
L^{*}=-A \frac{\partial}{\partial x}-B \frac{\partial}{\partial y}-\varepsilon\left(\left(C_{11} \frac{\partial}{\partial x}+C_{12} \frac{\partial}{\partial y}\right) \frac{\partial}{\partial x}+\left(C_{21} \frac{\partial}{\partial x}+C_{22} \frac{\partial}{\partial y}\right) \frac{\partial}{\partial y}\right),
$$

and we obtain the dual boundary conditions by applying the homogeneous primal boundary conditions to the boundary integral terms. By using (5), we can write (7) 
as

$$
\begin{aligned}
\int_{0}^{T} J(U) d t & =\int_{0}^{T}(\Theta, F) d t+\int_{0}^{T}\left(\Theta_{t}-L^{*} \Theta+G, U\right) d t \\
& +\int_{0}^{T} \int_{W} U^{T}\left(\left(A-H_{W}^{T}\right) \Theta+\varepsilon\left(C_{11} \Theta_{x}+C_{12} \Theta_{y}\right)\right) d y d t \\
& -\int_{0}^{T} \int_{E} U^{T}\left(\left(A+H_{E}^{T}\right) \Theta+\varepsilon\left(C_{11} \Theta_{x}+C_{12} \Theta_{y}\right)\right) d y d t \\
& +\int_{0}^{T} \int_{S} U^{T}\left(\left(B-H_{S}^{T}\right) \Theta+\varepsilon\left(C_{21} \Theta_{x}+C_{22} \Theta_{y}\right)\right) d x d t \\
& -\int_{0}^{T} \int_{N} U^{T}\left(\left(B+H_{N}^{T}\right) \Theta+\varepsilon\left(C_{21} \Theta_{x}+C_{22} \Theta_{y}\right)\right) d x d t .
\end{aligned}
$$

We introduce the dual time variable, $\tau=T-t$, and the function $\Theta$ has to satisfy the dual Navier-Stokes equations

$$
\Theta_{\tau}-A \Theta_{x}-B \Theta_{y}=\varepsilon\left(\left(C_{11} \Theta_{x}+C_{12} \Theta_{y}\right)_{x}+\left(C_{21} \Theta_{x}+C_{22} \Theta_{y}\right)_{y}\right)+G
$$

with the dual boundary conditions

$$
\begin{aligned}
& \left(A-H_{W}^{T}\right) \Theta+\varepsilon\left(C_{11} \Theta_{x}+C_{12} \Theta_{y}\right)=0, \quad\left(A+H_{E}^{T}\right) \Theta+\varepsilon\left(C_{11} \Theta_{x}+C_{12} \Theta_{y}\right)=0, \\
& \left(B-H_{S}^{T}\right) \Theta+\varepsilon\left(C_{21} \Theta_{x}+C_{22} \Theta_{y}\right)=0, \quad\left(B+H_{N}^{T}\right) \Theta+\varepsilon\left(C_{21} \Theta_{x}+C_{22} \Theta_{y}\right)=0,
\end{aligned}
$$

together with a homogeneous initial condition at $\tau=0$.

\section{Well-posed boundary conditions}

A necessary, but not sufficient, requirement on $H_{W, E, S, N}$ is that they give energy estimates. By applying the energy method to (3) and using the Green-Gauss formula 
for higher dimensional integration-by-parts we can write the energy as

$$
\begin{aligned}
\|U\|_{t}^{2} & =\int_{W} U^{T} A U d y-\varepsilon \int_{W}\left(U^{T}\left(C_{11} U_{x}+C_{12} U_{y}\right)+\left(U_{x}^{T} C_{11}+U_{y}^{T} C_{12}\right) U\right) d y \\
& -\int_{E} U^{T} A U d y-\varepsilon \int_{E}\left(U^{T}\left(C_{11} U_{x}+C_{12} U_{y}\right)+\left(U_{x}^{T} C_{11}+U_{y}^{T} C_{12}\right) U\right) d y \\
& +\int_{S} U^{T} B U d x-\varepsilon \int_{S}\left(U^{T}\left(C_{21} U_{x}+C_{22} U_{y}\right)+\left(U_{x}^{T} C_{21}+U_{y}^{T} C_{22}\right) U\right) d x \\
& -\int_{N} U^{T} B U d x-\varepsilon \int_{N}\left(U^{T}\left(C_{21} U_{x}+C_{22} U_{y}\right)+\left(U_{x}^{T} C_{21}+U_{y}^{T} C_{22}\right) U\right) d x \\
& -2 \varepsilon \int_{\Omega} \nabla U^{T} \cdot\left(C_{11} U_{x}+C_{12} U_{y}, C_{21} U_{x}+C_{22} U_{y}\right) d \Omega .
\end{aligned}
$$

The last term can be rewritten as

$$
\int_{\Omega} \nabla U^{T} \cdot\left(C_{11} U_{x}+C_{12} U_{y}, C_{21} U_{x}+C_{22} U_{y}\right) d \Omega=\int_{\Omega}\left(\nabla U^{T}\right) C(\nabla U)^{T} d \Omega
$$

where the $8 \times 8$ matrix

$$
C=\left[\begin{array}{ll}
C_{11} & C_{12} \\
C_{21} & C_{22}
\end{array}\right]
$$

is positive semi-definite under the standard assumption $2 \mu+3 \lambda \geq 0$ which is valid in ideal fluids $[15,10,35]$. Using the boundary conditions in (5), we can cancel the energy contribution from the viscous terms and get the final energy estimate as

$$
\begin{aligned}
\|U\|_{t}^{2}+2 \varepsilon \int_{\Omega}\left(\nabla U^{T}\right) C(\nabla U)^{T} d \Omega= & -\int_{W} U^{T}\left(-A+H_{W}+H_{W}^{T}\right) U d y-\int_{E} U^{T}\left(A+H_{E}+H_{E}^{T}\right) U d y \\
& -\int_{S} U^{T}\left(-B+H_{S}+H_{S}^{T}\right) U d x-\int_{N} U^{T}\left(B+H_{N}+H_{N}^{T}\right) U d x .
\end{aligned}
$$

It is clear that $H_{W, E, S, N}$ must be chosen such that

$$
\begin{array}{ll}
-A+H_{W}+H_{W}^{T} \geq 0, & A+H_{E}+H_{E}^{T} \geq 0, \\
-B+H_{S}+H_{S}^{T} \geq 0, & B+H_{N}+H_{N}^{T} \geq 0,
\end{array}
$$


in order to obtain a bounded energy growth and hence an energy estimate.

It is also necessary that the dual boundary conditions in (10) gives an energy estimate for the time-dependent dual problem (9). The energy method applied to (9) results, as before, in

$$
\begin{aligned}
\|\Theta\|_{\tau}^{2}+2 \varepsilon \int_{\Omega}\left(\nabla \Theta^{T}\right) C(\nabla \Theta)^{T} d \Omega & =-\int_{W} \Theta^{T}\left(-A+H_{W}+H_{W}^{T}\right) \Theta d y-\int_{E} \Theta^{T}\left(A+H_{E}+H_{E}^{T}\right) \Theta d y \\
& -\int_{S} \Theta^{T}\left(-B+H_{S}+H_{S}^{T}\right) \Theta d x-\int_{N} \Theta^{T}\left(B+H_{N}+H_{N}^{T}\right) \Theta d x
\end{aligned}
$$

and we can see that the same requirements for obtaining an energy estimate hold for the dual problem as for the primal problem.

To analyze the Euler equations we let $\varepsilon=0$ in which case (3) reduces to

$$
U_{t}+A U_{x}+B U_{y}=0
$$

with the boundary conditions

$$
H_{W} U=0, \quad H_{E} U=0, \quad H_{S} U=0, \quad H_{N} U=0 .
$$

The energy method applied to (13) results in

$$
\|U\|_{t}^{2}=\int_{W} U^{T} A U d y-\int_{E} U^{T} A U d y+\int_{S} U^{T} B U d x-\int_{N} U^{T} B U d x,
$$

to which we can add the boundary conditions in (14) to get

$$
\begin{aligned}
\|U\|_{t}^{2}= & -\int_{W} U^{T}\left(-A+H_{W}+H_{W}^{T}\right) U d y-\int_{E} U^{T}\left(A+H_{E}+H_{E}^{T}\right) U d y \\
& -\int_{S} U^{T}\left(-B+H_{S}+H_{S}^{T}\right) U d x-\int_{N} U^{T}\left(B+H_{N}+H_{N}^{T}\right) U d x,
\end{aligned}
$$

which is identical to (11), except for the dissipation term. The requirements (12) are thus sufficient to obtain an energy estimate also for the Euler equations.

The dual Euler equations are derived analogously to the dual Navier-Stokes equations, resulting in

$$
\Theta_{\tau}-A \Theta_{x}-B \Theta_{x}=0
$$


with the boundary conditions

$$
\left(A-H_{W}^{T}\right) \Theta=0, \quad\left(A+H_{E}^{T}\right) \Theta=0, \quad\left(B-H_{S}^{T}\right) \Theta=0, \quad\left(B+H_{N}^{T}\right) \Theta=0 .
$$

The energy method applied to (16), using (17), gives

$$
\begin{aligned}
\|\Theta\|_{\tau}^{2}= & -\int_{W} \Theta^{T}\left(-A+H_{W}+H_{W}^{T}\right) \Theta d y-\int_{E} \Theta^{T}\left(A+H_{E}+H_{E}^{T}\right) \Theta d y \\
& -\int_{S} \Theta^{T}\left(-B+H_{S}+H_{S}^{T}\right) \Theta d x-\int_{N} \Theta^{T}\left(B+H_{N}+H_{N}^{T}\right) \Theta d x
\end{aligned}
$$

which is identical to (15).

To obtain energy estimates for the primal and dual Navier-Stokes and Euler equations, it is hence sufficient that the matrices $H_{W, E, S, N}$ are constructed such that the requirements in (12) hold. Energy estimates are, however, not sufficient. It is also required that the correct number of boundary conditions are imposed to get well-posed problems. An operator which have an energy estimate with a minimal number of boundary conditions, such that existence is guaranteed, is called maximally semi-bounded and leads directly to well-posedness. See $[9,36]$. The number of boundary conditions can also be derived using the Laplace transform technique which is thoroughly discussed in $[9,41,17]$. A derivation is beyond the scope of this paper and we summarize the numbers required at each boundary under subsonic conditions in Table 1. For more information, see for example $[41,36]$.

Table 1: Number of boundary conditions required for the primal and dual Navier-Stokes and Euler equations under subsonic conditions with positive velocity components

\begin{tabular}{|l|c|c|c|c|}
\hline & \multicolumn{4}{|c|}{ Number of b.c. } \\
\hline Boundary & West & East & South & North \\
\hline Primal Navier-Stokes & 4 & 3 & 4 & 3 \\
Dual Navier-Stokes & 3 & 4 & 3 & 4 \\
Primal Euler & 3 & 1 & 3 & 1 \\
Dual Euler & 1 & 3 & 1 & 3 \\
\hline
\end{tabular}

\subsection{The east outflow boundary}

There are essentially three common (homogeneous) subsonic outflow boundary conditions for the primal Euler equations;

$$
u=0, \quad p=0, \quad \text { or } \quad A^{-} U=0,
$$


where $A^{-}=X \Lambda^{-} X^{-1}$ is constructed from the outgoing characteristics of $A$. See $[43,29,30]$ for more details. It is required that the matrix $H_{E}$ is constructed such that

$$
H_{E} U+\varepsilon\left(C_{11} U_{x}+C_{12} U_{y}\right)=0
$$

gives well-posed primal and dual Navier-Stokes equations, and as $\varepsilon \rightarrow 0$, the boundary conditions

$$
H_{E} U=0
$$

give well-posed primal and dual Euler equations. The characteristic boundary conditions are very well-suited for the Euler equations, but difficult to use as building blocks for the Navier-Stokes since too many boundary conditions will be imposed. This is because the viscous terms alone set 3 linearly independent boundary conditions, and $A^{-}$is a full matrix. Hence 4 boundary conditions will imposed instead of 3, making the problem ill-posed. Auxiliary matrices can to be constructed so that some linear dependence is removed in order to set the correct number of boundary conditions [43]. Specifying the velocity can remove the ill-posedness due to too many boundary conditions, but it is shown in the appendix that this is not a suitable boundary condition either.

Here we chose to construct $H_{E}$ such that the pressure is specified for the primal Euler equations. In this case, $H_{E}$ has to satisfy

1. The top row of $H_{E}$ is zero

2. The top row of $A+H_{E}^{T}$ is non-zero

3. $\operatorname{rank}\left(H_{E}\right)=1$

4. $\operatorname{rank}\left(A+H_{E}^{T}\right)=3$

5. $A+H_{E}+H_{E}^{T} \geq 0$

Requirements (1) and (2) set the correct number of boundary conditions for the primal and dual Navier-Stokes equations, requirements (3) and (4) set the correct number of boundary conditions for the primal and dual Euler equations, and requirement (5) gives energy estimates of both the primal and dual Navier-Stokes and Euler equations, respectively. 
Since we are working with the linearized equations, the pressure is specified in terms of the linearized equation of state (4). The boundary condition we consider for the primal Euler equations is $p=0$, or equivalently

$$
\bar{T} \rho+\bar{\rho} T=0 .
$$

By applying the energy method to the linearized Euler equations and using (19), it can be shown that

$$
\|U\|_{t}^{2} \leq 0
$$

and hence $p=0$ gives a well-posed problem. Considering all requirements, the matrix $H_{E}$ can be constructed on the form

$$
H_{E}=\left[\begin{array}{cccc}
0 & 0 & 0 & 0 \\
k_{2} & 0 & 0 & k_{2} \sqrt{\gamma-1} \\
k_{3} & 0 & 0 & k_{3} \sqrt{\gamma-1} \\
k_{4} & 0 & 0 & k_{4} \sqrt{\gamma-1}
\end{array}\right]
$$

where $k_{2,3,4}$ are to be determined. Note that

$$
H_{E} U=\frac{1}{\bar{\rho} \bar{c} \sqrt{\gamma}}\left[\begin{array}{c}
0 \\
k_{2}(\bar{T} \rho+\bar{\rho} T) \\
k_{3}(\bar{T} \rho+\bar{\rho} T) \\
k_{4}(\bar{T} \rho+\bar{\rho} T)
\end{array}\right]=\frac{\sqrt{\gamma}}{\bar{\rho} \bar{c}}\left[\begin{array}{c}
0 \\
k_{2} p \\
k_{3} p \\
k_{4} p
\end{array}\right]
$$

according to (2) and (4) and we are indeed specifying only one linearly dependent condition on the pressure. The matrix $H_{E}$ has zero top row and rank 1 , and hence satisfies conditions (1) and (3). In order to satisfy condition (5), we let

$$
k_{3}=k_{4}=0
$$

since then the eigenvalues $\lambda_{1,2,3,4}$ of $M_{E}=A+H_{E}+H_{E}^{T}$ can be directly computed as

$$
\lambda_{1,2}=\bar{u}, \quad \lambda_{3,4}=\bar{u} \pm \sqrt{\bar{c}^{2}+\gamma k_{2}^{2}+2 \bar{c} k_{2} \sqrt{\gamma}} .
$$

In order for $\lambda_{3,4}$, and thus $M_{E}$, to be positive semi-definite, it is required that

$$
k_{2}=\frac{\bar{c}-\sqrt{\bar{u}^{2}+\zeta_{2}}}{\sqrt{\gamma}},
$$


where $\zeta_{2} \leq 0$ is a free parameter. A direct computation of $A+H_{E}^{T}$ shows that condition (2) is satisfied, but condition (4) is not. The free parameter $\zeta_{2}$ can, however, be used. Gaussian elimination on $A+H_{E}^{T}$ shows that condition (4) is satisfied if, and only if, we chose

$$
\zeta_{2}=\frac{\bar{u}^{2}\left(\bar{u}^{2}-\bar{c}^{2}\right)}{\bar{c}^{2}} \leq 0
$$

The result is summarized in

Theorem 4.1. Let the matrix $H_{E}$ be given by

$$
H_{E}=\frac{\bar{u}^{2}-\bar{c}^{2}}{\bar{c} \sqrt{\gamma}}\left[\begin{array}{cccc}
0 & 0 & 0 & 0 \\
1 & 0 & 0 & \sqrt{\gamma-1} \\
0 & 0 & 0 & 0 \\
0 & 0 & 0 & 0
\end{array}\right]
$$

Then the boundary conditions

$$
H_{E} U+\varepsilon\left(C_{11} U_{x}+C_{12} U_{y}\right)=0
$$

are well-posed subsonic outflow conditions for the primal Navier-Stokes and, as $\varepsilon \rightarrow$ 0, also for the primal Euler equations, which specifies the pressure for the primal Euler equations. Moreover, they provide well-posed dual boundary conditions for the dual Navier-Stokes and Euler equations given in (10) and (17), respectively.

Proof. Requirements (1)-(4) can directly be seen to be satisfied. Requirement (5) is satisfied since the eigenvalues $\lambda_{1,2,3,4}$ of $M_{E}=A+H_{E}+H_{E}^{T}$ are given by

$$
\lambda_{1}=\bar{u}, \quad \lambda_{2}=\bar{u}, \quad \lambda_{3}=-\frac{\bar{u}(\bar{u}-\bar{c})}{\bar{c}}, \quad \lambda_{4}=\frac{\bar{u}(\bar{c}+\bar{u})}{\bar{c}}
$$

which are all positive since $\bar{u}<\bar{c}$ by assumption. Moreover, we can compute

$$
H_{E} U=\frac{\bar{u}^{2}-\bar{c}^{2}}{\bar{c} \sqrt{\gamma}}\left[\begin{array}{c}
0 \\
\frac{\bar{c}}{\bar{\rho} \sqrt{\gamma}} \rho+\frac{1}{\bar{c} \sqrt{\gamma}} T \\
0 \\
0
\end{array}\right]=\frac{\bar{u}^{2}-\bar{c}^{2}}{\bar{\rho} \bar{c}^{2}}\left[\begin{array}{l}
0 \\
p \\
0 \\
0
\end{array}\right]
$$

by using the fact that $\bar{c}^{2}=\bar{T}$ together with the linearized equation of state (4), which specifies the pressure. 


\subsection{West inflow boundary}

It is required that the matrix $H_{W}$ is constructed such that the boundary conditions

$$
H_{W} U-\varepsilon\left(C_{11} U_{x}+C_{12} U_{y}\right)=0
$$

give well-posed primal and dual Navier-Stokes equations, while

$$
H_{W} U=0
$$

give well-posed primal and dual Euler equations. Here, $H_{W}$ has to satisfy

1. The top row of $H_{W}$ is non-zero

2. The top row of $A-H_{W}^{T}$ is zero

3. $\operatorname{rank}\left(H_{W}\right)=3$

4. $\operatorname{rank}\left(A-H_{W}^{T}\right)=1$

5. $-A+H_{W}+H_{W}^{T} \geq 0$

Requirements (1)-(2) set the correct number of boundary conditions for the primal and dual Navier-Stokes equation and (3)-(4) set the correct number of boundary conditions for the primal and dual Euler equations. Requirement (5) provides energy estimates for all primal and dual equations.

At the east outflow boundary, we used the pressure as the boundary condition for the primal Euler equation, which required only 1 boundary condition. Here, it is the dual Euler equation which require only one boundary condition. However, the dual variables have no clear physical meaning and there is no equation of state to relate the different dual variables. We will use the energy method to derive a suitable boundary condition for the dual Euler equation, which will be the analogous to the pressure for the primal Euler equations. The energy method applied to (16) results in

$$
\|\Theta\|_{\tau}^{2}=-\int_{W} \Theta^{T} A \Theta d y,
$$

when only considering the west boundary terms. By expanding the quadratic term in (21) and completing squares we get

$$
\|\Theta\|_{\tau}^{2} \leq-2 \frac{\bar{c}}{\sqrt{\gamma}} \theta_{2}\left(\theta_{1}+\sqrt{\gamma-1} \theta_{4}\right),
$$

and there are three possible boundary conditions which give an energy estimate; 
I. $\theta_{2}=0$

II. $\theta_{1}+\sqrt{\gamma-1} \theta_{4}=0$

III. Linear combinations of the above

The first option is the analogue of specifying the velocity for the dual Euler equation and is not suitable for reasons given in the appendix. The third option can be used to obtain the characteristic boundary conditions $[43,29]$. We will base the structure of $H_{W}$ on the second option which is the analogue of specifying the pressure for the primal Euler equations. It is then required that $H_{W}$ is constructed so that

$$
A-H_{W}^{T}=\left[\begin{array}{cccc}
0 & 0 & 0 & 0 \\
k_{2} & 0 & 0 & k_{2} \sqrt{\gamma-1} \\
k_{3} & 0 & 0 & k_{3} \sqrt{\gamma-1} \\
k_{4} & 0 & 0 & k_{4} \sqrt{\gamma-1}
\end{array}\right]
$$

or equivalently

$$
H_{W}=\left[\begin{array}{cccc}
\bar{u} & \frac{\bar{c}}{\sqrt{\gamma}}-k_{2} & -k_{3} & -k_{4} \\
\bar{c} & \bar{u} & 0 & \bar{c} \sqrt{\frac{\gamma-1}{\gamma}} \\
0 & 0 & \bar{u} & 0 \\
0 & \overline{\sqrt{\gamma}} \sqrt{\frac{\gamma-1}{\gamma}}-k_{2} \sqrt{\gamma-1} & -k_{3} \sqrt{\gamma-1} & \bar{u}-k_{4} \sqrt{\gamma-1}
\end{array}\right]
$$

It is seen that $H_{W}$ satisfies requirements (1), (2), and (4). To satisfy requirement (5), we let $k_{3}=k_{4}=0$ since then the eigenvalues of the matrix $M_{W}=-A+H_{W}+H_{W}^{T}$ can be directly computed as

$$
\lambda_{1,2}=\bar{u}, \quad \lambda_{3,4}=\bar{u} \pm \sqrt{\bar{c}^{2}+\gamma k_{2}^{2}-2 \bar{c} k_{2} \sqrt{\gamma}}
$$

In order for both $\lambda_{3,4}$, and hence $M_{W}$, to be positive, it is required that

$$
k_{2}=\frac{\bar{c}-\sqrt{\bar{u}^{2}-\zeta_{2}}}{\sqrt{\gamma}}
$$

where $\zeta_{2} \leq 0$ is a free parameter. To satisfy (3) which is the last requirement, one can show by Gaussian elimination that $H_{W}$ have rank 3 if, and only if, we let

$$
\zeta_{2}=\frac{\bar{u}^{2}\left(\bar{u}^{2}-\bar{c}^{2}\right)}{\bar{c}^{2}} \leq 0
$$

The results are summarized in 
Theorem 4.2. Let the matrix $H_{W}$ be given by

$$
H_{W}=\left[\begin{array}{cccc}
\bar{u} & \frac{\bar{u}^{2}}{\bar{c} \sqrt{\gamma}} & 0 & 0 \\
\bar{c} & \bar{u} & 0 & \bar{c} \sqrt{\frac{\gamma-1}{\gamma}} \\
0 & 0 & \bar{u} & 0 \\
0 & \frac{\bar{u}^{2}}{\bar{c}} \sqrt{\frac{\gamma-1}{\gamma}} & 0 & \bar{u}
\end{array}\right] .
$$

Then the boundary conditions

$$
H_{W} U-\varepsilon\left(C_{11} U_{x}+C_{12} U_{y}\right)=0
$$

are well-posed subsonic inflow conditions for the primal Navier-Stokes equations, and as $\varepsilon \rightarrow 0$, also for the primal Euler equations. Moreover, they provide well-posed dual boundary conditions for the dual Navier-Stokes and Euler equations given in (10) and (17), respectively.

Proof. The requirements (1), (2), and (4) can directly be seen. Requirement (3) can be seen by performing Gaussian elimination on $H_{W}$. Requirement (5) is satisfied since the eigenvalues $\lambda_{1,2,3,4}$ of $M_{W}=-A+H_{W}+H_{W}^{T}$ can be directly computed as

$$
\lambda_{1}=\bar{u}, \quad \lambda_{2}=\bar{u}, \quad \lambda_{3}=-\frac{\bar{u}(\bar{u}-\bar{c})}{\bar{c}}, \quad \lambda_{4}=\frac{\bar{u}(\bar{u}+\bar{c})}{\bar{c}}
$$

which are all positive since $\bar{u}<\bar{c}$ by assumption.

Note that there are no free parameters left in the construction of $H_{W}$. The boundary conditions (20) hence uniquely determine the subsonic inflow boundary conditions for the primal Euler equations. By explicitly computing $H_{W} U$, we can write (20) in component form as

$$
\begin{aligned}
\frac{\bar{u}}{\bar{\rho} \bar{c} \sqrt{\gamma}}(\bar{T} \rho+\bar{\rho} \bar{u} u) & =0, \\
\bar{\rho}^{-1} p+\bar{u} u & =0, \\
\bar{u} v & =0, \\
\overline{\bar{u}}((\gamma-1) \bar{u} u+T) & =0 .
\end{aligned}
$$

Note that there are only three linearly independent equations above since $\operatorname{rank}\left(H_{W}\right)=3$. These results can be compared with the results in i.e. [29]. 


\subsection{North and south boundaries}

The same arguments as for the east/west outflow/inflow boundaries can be applied to the north/south outflow/inflow boundaries. The only thing that needs to be done is to replace the matrix $A$ by $B, H_{W}$ by $H_{S}, H_{E}$ by $H_{N}$, and repeat the procedure. The resulting matrices $H_{N}$ and $H_{S}$ are given in

Theorem 4.3. Let the matrices $H_{N}$ and $H_{S}$ be given by

$$
H_{N}=\frac{\bar{v}^{2}-\bar{c}^{2}}{\bar{c} \sqrt{\gamma}}\left[\begin{array}{cccc}
0 & 0 & 0 & 0 \\
0 & 0 & 0 & 0 \\
1 & 0 & 0 & \sqrt{\gamma-1} \\
0 & 0 & 0 & 0
\end{array}\right], H_{S}=\left[\begin{array}{cccc}
\bar{v} & 0 & \frac{\bar{v}^{2}}{\bar{c} \sqrt{\gamma}} & 0 \\
0 & \bar{v} & 0 & 0 \\
\bar{c} & 0 & \bar{v} & \bar{c} \sqrt{\frac{\gamma-1}{\gamma}} \\
\frac{\sqrt{\gamma}}{0} & 0 & \frac{\bar{v}^{2}}{\bar{c}} \sqrt{\frac{\gamma-1}{\gamma}} & \bar{v}
\end{array}\right] \text {. }
$$

Then the boundary conditions

$$
H_{S} U-\varepsilon\left(C_{21} U_{x}+C_{22} U_{y}\right)=0, \quad H_{N} U+\varepsilon\left(C_{21} U_{x}+C_{22} U_{y}\right)=0
$$

are well-posed boundary conditions for the primal Navier-Stokes equations, and as $\varepsilon \rightarrow 0$, also for the primal Euler equations. For $\varepsilon=0$, the pressure is specified as the outflow boundary condition for the Euler equations. Moreover, they provide well-posed dual boundary conditions for the dual Navier-Stokes and Euler equations given in (10) and (17), respectively.

Proof. By following the procedure for the west/east boundaries, the matrices set the correct number of boundary conditions for the primal and dual Navier-Stokes equations when $\varepsilon \neq 0$, and for the primal and dual Euler equations when $\varepsilon=0$. The symmetric boundary matrices in the energy estimate,

$$
M_{N}=B+H_{N}+H_{N}^{T}, \quad M_{S}=-B+H_{S}+H_{S}^{T}
$$

have the same eigenvalues, given by

$$
\lambda_{1}=\bar{v}, \quad \lambda_{2}=\bar{v}, \quad \lambda_{3}=-\frac{\bar{v}(\bar{v}-\bar{c})}{\bar{c}}, \quad \lambda_{4}=\frac{\bar{v}(\bar{c}+\bar{v})}{\bar{c}},
$$

which are all positive since $\bar{v}<\bar{c}$ by assumption. 


\section{Summation-by-parts operators}

An SBP-operator is in essence a central finite difference matrix which have been modified close to the boundaries to become one-sided. The SBP-operator $D_{1}$ can be decomposed as $D_{1}=P^{-1} Q$, where $P$ is positive symmetric definite and $Q+Q^{T}=$ $\operatorname{diag}[-1,0, \ldots, 0,1]$. All details can be found in [39]. The second derivative operator $D_{2}$ can be obtained as $D_{2}=D_{1} D_{1}$ which results in a wide operator, or compactly as $D_{2}=P^{-1}(-A+B S)$. See [24] for details on the second derivative operator. In this paper, we have the equations in conservative or flux form and hence a second derivative operator is not required.

The extension to multiple dimensions is done by using the Kronecker product. The Kronecker product is defined as

$$
(A \otimes B)=\left[\begin{array}{ccc}
a_{11} B & \cdots & a_{1 n} B \\
\vdots & \ddots & \vdots \\
a_{m 1} B & \cdots & a_{m n} B
\end{array}\right]
$$

for any matrices $A \in \mathbb{R}^{m \times n}$ and $B \in \mathbb{R}^{p \times q}$. Two properties which will be used are the mixed product property,

$$
(A \otimes B)(C \otimes D)=(A C \otimes D B)
$$

if the usual matrix products are defined, and

$$
(A \otimes B)^{-1, T}=\left(A^{-1, T} \otimes B^{-1, T}\right)
$$

for the inverse and transpose, if the usual matrix inverses are defined. We define the following operators for the multi-dimensional extension;

$$
\begin{aligned}
D_{x} & =\left(P_{x}^{-1} Q_{x} \otimes I_{y}\right), & D_{y} & =\left(I_{x} \otimes P_{y}^{-1} Q_{y}\right), \\
\bar{D}_{x} & =\left(P_{x}^{-1} Q_{x} \otimes I_{y} \otimes I_{4}\right), & \bar{D}_{y} & =\left(I_{x} \otimes P_{y}^{-1} Q_{y} \otimes I_{4}\right), \\
I & =\left(I_{x} \otimes I_{y}\right), & P & =\left(P_{x} \otimes P_{y}\right) . \\
E_{W} & =\operatorname{diag}[1,0, \ldots, 0], & E_{E} & =\operatorname{diag}[0, \ldots, 0,1], \\
E_{S} & =\operatorname{diag}[1,0, \ldots, 0], & E_{N} & =\operatorname{diag}[0, \ldots, 0,1],
\end{aligned}
$$

The $x, y$ subscripts indicate differentiation in the corresponding coordinate direction, and the $E, W, S, N$ subscripts indicate that the operator operates on the corresponding boundary only. $I_{x, y}$ are the identity matrices in the corresponding coordinate direction. Note that there can be different number of grid points in the difference coordinate directions and hence the sizes of the matrices can differ. 


\section{Discretization, stability, and dual consistency}

To perform a stability analysis, we discretize the linear and symmetric system (3) with the boundary conditions in (5). In the computations, however, the non-linear equations (1) is used and the system have been transformed to its conservative form.

An SBP-SAT discretization of (3) can be written as

$$
\begin{aligned}
& \frac{d}{d t} U_{h}+\left(D_{x} \otimes A\right) U_{h}+\left(D_{y} \otimes B\right) U_{h} \\
& -\varepsilon \bar{D}_{x}\left(\left(D_{x} \otimes C_{11}\right) U_{h}+\left(D_{y} \otimes C_{12}\right) U_{h}\right) \\
& -\varepsilon \bar{D}_{y}\left(\left(D_{x} \otimes C_{21}\right) U_{h}+\left(D_{y} \otimes C_{22}\right) U_{h}\right) \\
& =\left(P_{x}^{-1} E_{W} \otimes I_{y} \otimes \Sigma_{W}\right)\left(\left(I \otimes H_{W}\right) U_{h}-\varepsilon\left(\left(D_{x} \otimes C_{11}\right) U_{h}+\left(D_{y} \otimes C_{12}\right) U_{h}\right)\right) \\
& +\left(P_{x}^{-1} E_{E} \otimes I_{y} \otimes \Sigma_{E}\right)\left(\left(I \otimes H_{E}\right) U_{h}+\varepsilon\left(\left(D_{x} \otimes C_{11}\right) U_{h}+\left(D_{y} \otimes C_{12}\right) U_{h}\right)\right) \\
& +\left(I_{x} \otimes P_{y}^{-1} E_{S} \otimes \Sigma_{S}\right)\left(\left(I \otimes H_{S}\right) U_{h}-\varepsilon\left(\left(D_{x} \otimes C_{21}\right) U_{h}+\left(D_{y} \otimes C_{22}\right) U_{h}\right)\right) \\
& +\left(I_{x} \otimes P_{y}^{-1} E_{N} \otimes \Sigma_{N}\right)\left(\left(I \otimes H_{N}\right) U_{h}+\varepsilon\left(\left(D_{x} \otimes C_{21}\right) U_{h}+\left(D_{y} \otimes C_{22}\right) U_{h}\right)\right)
\end{aligned}
$$

where the terms before the equality sign approximate the equations, and the terms after impose the boundary conditions (5). The matrices $\Sigma_{W, E, S, N} \in \mathbb{R}^{4 \times 4}$ have to be determined such that the scheme is stable. These are given in

Theorem 6.1. The scheme (22) is energy stable when choosing

$$
\Sigma_{W}=\Sigma_{E}=\Sigma_{S}=\Sigma_{N}=-I_{4},
$$

where $I_{4}$ is the $4 \times 4$ identity matrix. 
Proof. The energy method applied to (22) results in

$$
\begin{aligned}
\left\|U_{h}\right\|_{t}^{2}+\mathrm{DI}\left(U_{h}\right) & =U_{h}^{T}\left(E_{W} \otimes P_{y} \otimes\left(A+\Sigma_{W} H_{W}+H_{W}^{T} \Sigma_{W}^{T}\right)\right) U_{h} \\
& +U_{h}^{T}\left(E_{E} \otimes P_{y} \otimes\left(-A+\Sigma_{E} H_{E}+H_{E}^{T} \Sigma_{E}^{T}\right)\right) U_{h} \\
& +U_{h}^{T}\left(P_{x} \otimes E_{S} \otimes\left(B+\Sigma_{S} H_{S}+H_{S}^{T} \Sigma_{S}^{T}\right)\right) U_{h} \\
& +U_{h}^{T}\left(P_{x} \otimes E_{N} \otimes\left(-B+\Sigma_{N} H_{N}+H_{N}^{T} \Sigma_{N}^{T}\right)\right) U_{h} \\
& -2 \varepsilon U_{h}^{T}\left(E_{W} P_{x}^{-1} Q_{x} \otimes P_{y} \otimes\left(C_{11}+\Sigma_{W} C_{11}\right)\right) U_{h} \\
& +2 \varepsilon U_{h}^{T}\left(E_{E} P_{x}^{-1} Q_{x} \otimes P_{y} \otimes\left(C_{11}+\Sigma_{W} C_{11}\right)\right) U_{h} \\
& -2 \varepsilon U_{h}^{T}\left(E_{W} \otimes Q_{y} \otimes\left(C_{12}+\Sigma_{E} C_{12}\right)\right) U_{h} \\
& +2 \varepsilon U_{h}^{T}\left(E_{E} \otimes Q_{y} \otimes\left(C_{12}+\Sigma_{E} C_{12}\right)\right) U_{h} \\
& -2 \varepsilon U_{h}^{T}\left(Q_{x} \otimes E_{S} \otimes\left(C_{21}+\Sigma_{S} C_{21}\right)\right) U_{h} \\
& +2 \varepsilon U_{h}^{T}\left(Q_{x} \otimes E_{N} \otimes\left(C_{21}+\Sigma_{N} C_{21}\right)\right) U_{h} \\
& -2 \varepsilon U_{h}^{T}\left(P_{x} \otimes E_{S} P_{y}^{-1} Q_{y} \otimes\left(C_{22}+\Sigma_{S} C_{22}\right)\right) U_{h} \\
& +2 \varepsilon U_{h}^{T}\left(P_{x} \otimes E_{N} P_{y}^{-1} Q_{y} \otimes\left(C_{22}+\Sigma_{N} C_{22}\right)\right) U_{h},
\end{aligned}
$$

where the term $\operatorname{DI}\left(U_{h}\right)$ can be written as

$$
\operatorname{DI}\left(U_{h}\right)=2 \varepsilon\left[\begin{array}{c}
\bar{D}_{x} U_{h} \\
\bar{D}_{y} U_{h}
\end{array}\right]^{T}\left[\begin{array}{ll}
\left(P \otimes C_{11}\right) & \left(P \otimes C_{12}\right) \\
\left(P \otimes C_{21}\right) & \left(P \otimes C_{22}\right)
\end{array}\right]\left[\begin{array}{c}
\bar{D}_{x} U_{h} \\
\bar{D}_{y} U_{h}
\end{array}\right]
$$

and is purely dissipative $[36,43,2]$. By the choices in (23), the expression (24) simplifies to

$$
\begin{aligned}
\left\|U_{h}\right\|_{t}^{2}+\mathrm{DI}\left(U_{h}\right)= & -U_{h}^{T}\left(E_{W} \otimes P_{y} \otimes\left(-A+H_{W}+H_{W}^{T}\right)\right) U_{h} \\
& -U_{h}^{T}\left(E_{E} \otimes P_{y} \otimes\left(A+H_{E}+H_{E}^{T}\right)\right) U_{h} \\
& -U_{h}^{T}\left(P_{x} \otimes E_{S} \otimes\left(-B+H_{S}+H_{S}^{T}\right)\right) U_{h} \\
& -U_{h}^{T}\left(P_{x} \otimes E_{N} \otimes\left(B+H_{N}+H_{N}^{T}\right)\right) U_{h},
\end{aligned}
$$

where the matrices $H_{W, E, S, N}$ are constructed such that (12) holds and hence

$$
\left\|U_{h}\right\|_{t}^{2}+\mathrm{DI}\left(U_{h}\right) \leq 0
$$

Thus an energy estimate have been obtained and the scheme (22) is energy stable.

The discrete dual operator $L_{h}^{*}$ is defined as the operator satisfying

$$
\left(v_{h}, L_{h} u_{h}\right)_{h}=\left(L_{h}^{*} v_{h}, u_{h}\right)_{h},
$$


where the inner product in the SBP framwork is defined as

$$
\left(v_{h}, u_{h}\right)_{h}=v_{h}^{T}\left(P \otimes I_{4}\right) u_{h}
$$

for all grid functions $u_{h}, v_{h}$. Hence the discrete dual operator can be explicitly computed as

$$
L_{h}^{*}=\left(P \otimes I_{4}\right)^{-1} L_{h}^{T}\left(P \otimes I_{4}\right) .
$$

For the scheme (22) to be dual consistent, it is required that the discrete dual operator $L_{h}^{*}$ approximates the dual operator $L^{*}$ in (8), together with the dual boundary conditions in (10). The main result of this paper can be summarized in

Theorem 6.2. The discretization (22) is energy stable and dual consistent with the choice of penalty coefficients given in (23).

Proof. Energy stability has already been proven, and we must show that the scheme (22) together with the coefficients in (23) is dual consistent. To prove this, it is convenient to write (22), using (23), in operator form as

$$
\frac{d}{d t} U_{h}+L_{h} U_{h}=0
$$

where

$$
\begin{aligned}
& L_{h}=\left(D_{x} \otimes A\right)+\left(D_{y} \otimes B\right) \\
& -\varepsilon \bar{D}_{x}\left(\left(D_{x} \otimes C_{11}\right)+\left(D_{y} \otimes C_{12}\right)\right) \\
& -\varepsilon \bar{D}_{y}\left(\left(D_{x} \otimes C_{21}\right)+\left(D_{y} \otimes C_{22}\right)\right) \\
& +\left(P_{x}^{-1} E_{W} \otimes I_{y} \otimes I_{4}\right)\left(\left(I \otimes H_{W}\right)-\varepsilon\left(\left(D_{x} \otimes C_{11}\right)+\left(D_{y} \otimes C_{12}\right)\right)\right) \\
& +\left(P_{x}^{-1} E_{E} \otimes I_{y} \otimes I_{4}\right)\left(\left(I \otimes H_{E}\right)+\varepsilon\left(\left(D_{x} \otimes C_{11}\right)+\left(D_{y} \otimes C_{12}\right)\right)\right) \\
& +\left(I_{x} \otimes P_{y}^{-1} E_{S} \otimes I_{4}\right)\left(\left(I \otimes H_{S}\right)-\varepsilon\left(\left(D_{x} \otimes C_{21}\right)+\left(D_{y} \otimes C_{22}\right)\right)\right) \\
& +\left(I_{x} \otimes P_{y}^{-1} E_{N} \otimes I_{4}\right)\left(\left(I \otimes H_{N}\right)+\varepsilon\left(\left(D_{x} \otimes C_{21}\right)+\left(D_{y} \otimes C_{22}\right)\right)\right) .
\end{aligned}
$$

To find the discrete dual problem we add a forcing function $F$ to (27),

$$
\begin{aligned}
\frac{d}{d t} U_{h}+L_{h} U_{h} & =F, \\
J_{h}\left(U_{h}\right) & =\left(G, U_{h}\right)_{h},
\end{aligned}
$$

together with a linear functional, where $G$ a weight function. Analogously to the continuous case, we seek a function $\Theta_{h}$ such that $\int_{0}^{T} J_{h}\left(U_{h}\right) d t=\int_{0}^{T}\left(\Theta_{h}, F\right)_{h} d t$. Integration 
by parts gives

$$
\begin{aligned}
\int_{0}^{T} J_{h}\left(U_{h}\right) d t & =\int_{0}^{T}\left(G, U_{h}\right)_{h} d t-\int_{0}^{T}\left(\Theta_{h}, \frac{d}{d t} U_{h}+L_{h} U_{h}-F\right)_{h} d t \\
& =\int\left(\Theta_{h}, F\right)_{h} d t+\int_{0}^{T}\left(\frac{d}{d t} \Theta_{h}-L_{h}^{*} \Theta_{h}+G, U_{h}\right)_{h} d t
\end{aligned}
$$

where

$$
\begin{aligned}
L_{h}^{*} & =-\left(D_{x} \otimes A\right)-\left(D_{y} \otimes B\right) \\
& -\varepsilon\left(P_{x}^{-1} Q_{q} \otimes I_{y} \otimes I_{4}\right)\left(\left(D_{x} \otimes C_{11}\right)+\left(D_{y} \otimes C_{12}\right)\right) \\
& -\varepsilon\left(I_{y} \otimes P_{y}^{-1} Q_{y} \otimes I_{4}\right)\left(\left(D_{x} \otimes C_{21}\right)+\left(D_{y} \otimes C_{22}\right)\right) \\
& -\left(P_{x}^{-1} E_{W} \otimes I_{y} \otimes I_{4}\right)\left(\left(I \otimes\left(A-H_{W}^{T}\right)\right)+\varepsilon\left(\left(D_{x} \otimes C_{11}\right)+\left(D_{y} \otimes C_{12}\right)\right)\right) \\
& +\left(P_{x}^{-1} E_{E} \otimes I_{y} \otimes I_{4}\right)\left(\left(I \otimes\left(A+H_{E}^{T}\right)\right)+\varepsilon\left(\left(D_{x} \otimes C_{11}\right)+\left(D_{y} \otimes C_{12}\right)\right)\right) \\
& -\left(I_{x} \otimes P_{y}^{-1} E_{S} \otimes I_{4}\right)\left(\left(I \otimes\left(B-H_{S}^{T}\right)\right)+\varepsilon\left(\left(D_{x} \otimes C_{21}\right)+\left(D_{y} \otimes C_{22}\right)\right)\right) \\
& +\left(I_{x} \otimes P_{y}^{-1} E_{N} \otimes I_{4}\right)\left(\left(I \otimes\left(B+H_{N}^{T}\right)\right)+\varepsilon\left(\left(D_{x} \otimes C_{21}\right)+\left(D_{y} \otimes C_{22}\right)\right)\right) .
\end{aligned}
$$

The function $\Theta_{h}$ has thus to satisfy the discrete dual problem

$$
\frac{d}{d \tau} \Theta_{h}+L_{h}^{*} \Theta_{h}=G
$$

where $\tau=T-t$. We can see that the six first terms in (28) approximate the continuous dual operator (8), while the last four terms imposes the dual boundary conditions in (10). The discrete dual operator is thus a consistent approximation of the dual problem and the scheme (22) is hence dual consistent, as well as energy stable, with the choices in (23).

Remark 6.1. Note that the requirements for stability are sufficient for dual consistency. The other direction does also hold - dual consistency is sufficient for stability in this case. Stability and dual consistency are hence equivalent for the type of boundary conditions in (5). That is not a general fact. It was shown in $[12,3]$ that dual consistency requires a subset of the stability conditions. A stable discretization can hence be dual inconsistent. On the contrary, one can also construct a dual consistent discretization which is unstable. 
Recall that the primal and dual equations have the same energy estimate in the continuous case. This holds also for the discretized equations. The energy method applied to the time-dependent discrete dual problem (29) results in

$$
\begin{aligned}
\left\|\Theta_{h}\right\|_{\tau}^{2}+\mathrm{DI}\left(\Theta_{h}\right)= & -\Theta_{h}^{T}\left(E_{W} \otimes P_{y} \otimes\left(-A+H_{W}+H_{W}^{T}\right)\right) \Theta_{h} \\
& -\Theta_{h}^{T}\left(E_{E} \otimes P_{y} \otimes\left(A+H_{E}+H_{E}^{T}\right)\right) \Theta_{h} \\
& -\Theta_{h}^{T}\left(P_{x} \otimes E_{S} \otimes\left(-B+H_{S}+H_{S}^{T}\right)\right) \Theta_{h} \\
& -\Theta_{h}^{T}\left(P_{x} \otimes E_{N} \otimes\left(B+H_{N}+H_{N}^{T}\right)\right) \Theta_{h},
\end{aligned}
$$

which is identical to the energy estimate of the discrete primal problem (25). Hence the discretization of the dual problem is also energy stable. This can open for a efficient method for simultaneous solution of the dual problem since much of the structure for the primal problem can be re-used. This chould be of interest for example when computing gradients in gradient optimization problems, or to further enhance functional superconverence as descripbed in [11].

To obtain approximations of the primal and dual Euler equations, we simply let $\varepsilon=0$. The construction of the continuous boundary conditions ensure that the resulting schemes are both energy stable and dual consistent. With $\varepsilon=0$, the scheme (22) reduces to

with

$$
\frac{d}{d t} U_{h}+L_{h} U_{h}=0
$$

$$
\begin{aligned}
L_{h} & =\left(D_{x} \otimes A\right)+\left(D_{y} \otimes B\right) \\
& +\left(P_{x}^{-1} E_{W} \otimes I_{y} \otimes I_{4}\right)\left(\left(I \otimes H_{W}\right)+\left(P_{x}^{-1} E_{E} \otimes I_{y} \otimes I_{4}\right)\left(\left(I \otimes H_{E}\right)\right.\right. \\
& +\left(I_{x} \otimes P_{y}^{-1} E_{S} \otimes I_{4}\right)\left(\left(I \otimes H_{S}\right)+\left(I_{x} \otimes P_{y}^{-1} E_{N} \otimes I_{4}\right)\left(\left(I \otimes H_{N}\right)\right.\right.
\end{aligned}
$$

and the energy estimate is given by

$$
\begin{aligned}
\left\|U_{h}\right\|_{t}^{2}= & -U_{h}^{T}\left(E_{W} \otimes P_{y} \otimes\left(-A+H_{W}+H_{W}^{T}\right)\right) U_{h} \\
& -U_{h}^{T}\left(E_{E} \otimes P_{y} \otimes\left(A+H_{E}+H_{E}^{T}\right)\right) U_{h} \\
& -U_{h}^{T}\left(P_{x} \otimes E_{S} \otimes\left(-B+H_{S}+H_{S}^{T}\right)\right) U_{h} \\
& -U_{h}^{T}\left(P_{x} \otimes E_{N} \otimes\left(B+H_{N}+H_{N}^{T}\right)\right) U_{h},
\end{aligned}
$$

which is identical to (25) except for the dissipation from the viscous terms. The discrete dual operator can be directly computed according to (26) as

$$
\begin{aligned}
L_{h}^{*}= & -\left(D_{x} \otimes A\right)-\left(D_{y} \otimes B\right) \\
& -\left(P_{x}^{-1} E_{W} \otimes I_{y} \otimes I_{4}\right)\left(\left(I \otimes\left(A-H_{W}^{T}\right)\right)+\left(P_{x}^{-1} E_{E} \otimes I_{y} \otimes I_{4}\right)\left(\left(I \otimes\left(A+H_{E}^{T}\right)\right)\right.\right. \\
& -\left(I_{x} \otimes P_{y}^{-1} E_{S} \otimes I_{4}\right)\left(\left(I \otimes\left(B-H_{S}^{T}\right)\right)+\left(I_{x} \otimes P_{y}^{-1} E_{N} \otimes I_{4}\right)\left(\left(I \otimes\left(B+H_{N}^{T}\right)\right),\right.\right.
\end{aligned}
$$


which gives a consistent approximation of the dual Euler equations (16) with the dual boundary conditions in (17). Similarly, the energy method applied to the discrete dual Euler equations

$$
\frac{d}{d t} \Theta_{\tau}+L_{h}^{*} \Theta_{h}=0
$$

results in

$$
\begin{aligned}
\left\|\Theta_{h}\right\|_{\tau}^{2}= & -\Theta_{h}^{T}\left(E_{W} \otimes P_{y} \otimes\left(-A+H_{W}+H_{W}^{T}\right)\right) \Theta_{h} \\
& -\Theta_{h}^{T}\left(E_{E} \otimes P_{y} \otimes\left(A+H_{E}+H_{E}^{T}\right)\right) \Theta_{h} \\
& -\Theta_{h}^{T}\left(P_{x} \otimes E_{S} \otimes\left(-B+H_{S}+H_{S}^{T}\right)\right) \Theta_{h} \\
& -\Theta_{h}^{T}\left(P_{x} \otimes E_{N} \otimes\left(B+H_{N}+H_{N}^{T}\right)\right) \Theta_{h}
\end{aligned}
$$

which is identical to $(30)$.

\subsection{Remarks on an implementation}

Energy stability and dual consistency are derived using integration by parts and the summation-by-parts properties of the difference operators. This requires that the equations are linearized and symmetrized. The linear theory is, of course, not valid for strongly non-linear problems involving shocks. It is, however, valid for nonlinear problems where those phenomena are excluded, see [18, 40]. Furthermore, it has been shown in many papers that energy stability is sufficient for a wide range of realistic applications. See for example [27, 44, 25, 14, 33].

The matrices $H_{W, E, S, N}$ that we have derived so far are based on linearizations. In the non-linear setting, one of the matrices $H_{W, E, S, N}$ has to be constructed for each grid point along the boundary and $\bar{u}, \bar{v}$ are taken as the actual velocity values in that grid point. It is possible, however, that either $u$ and/or $v$ is negative, for example if a vortex passes through the boundary. New matrices $H_{W, E, S, N}$ need then to be constructed. The relations are fortunately easily derived due to the symmetry of the problem. Denote by $H_{W, E}^{+}$the case where $\bar{u}>0$ and by $H_{S, N}^{+}$the case where $\bar{v}>0$. Denote by $H_{W, E}^{-}$the case where $\bar{u}<0$ and by $H_{S, N}^{-}$the case where $\bar{v}<0$. Then we have the following relations;

$$
H_{E}^{-}=-H_{W}^{+}, \quad H_{W}^{-}=-H_{E}^{+}, \quad H_{S}^{-}=-H_{N}^{+}, \quad H_{N}^{-}=-H_{S}^{+} .
$$

The penalty matrices given in theorem 6.1 remain the same and no other changes have to be made to the numerical scheme.

In a computer implementation of the non-linear problem on conservative form, one must loop through all grid points along the boundaries where boundary conditions are needed, and construct the matrices depending on if the velocity component 
is positive or negative. After the matrices have been constructed, they are transformed to conservative form from the symmetric form in which they were derived. The transformation matrices from symmetric to primitive can be found in [1], and the transformation matrices from primitive to conservative are described in [37].

\section{Numerical results}

The theory of functional superconvergence is based on linear problems with constant coefficients and linear integral functionals. For these problems the theoretical and numerical results are in good agreement $[13,12,11,3,4]$. Here we will apply the linear theory to the fully non-linear Navier-Stokes and Euler equations in conservation form to see whether or not the theory holds also in this case.

To verify the order of convergence we use the method of manufactured solutions $[38,21]$. We chose the solution

$$
\begin{aligned}
\rho & =1+\frac{1}{2} \sin (\pi(x-y)-t), & u & =\frac{1}{2} \cos (\pi x+y-t), \\
v & =\frac{1}{2} \sin (x+\pi y-t), & p & =1+\frac{1}{2} \cos (\pi(x-y)-t) \sin (\pi(x+y)-t) .
\end{aligned}
$$

which is inserted into (1) to compute a forcing function, $H$. We are hence solving the modified Navier-Stokes (or Euler if $\varepsilon=0$ ) equations

$$
q_{t}+F_{x}^{I}+G_{y}^{I}-\varepsilon\left(F_{x}^{V}+G_{y}^{V}\right)=H(x, y, t) .
$$

The addition of the forcing function does not alter the well-posedness due to the principle of Duhamel [9]. The manufactured solution is used to create initial- and boundary data and we measure the rate of convergence towards this solution. The manufactured solution has been chosen so that all boundaries contains both inflow and outflow at the same time. We perform the time integration using the classical 4th-order Runge-Kutta until time $t=0.1$ using 1000 time steps. The time step has been chosen sufficiently small so that the time integration errors are negliable compared to the spatial discretization errors.

There are SBP operators available with internal accuracy of $2 p=2,4,6$, and 8 . Remember that with a diagonal norm, the boundary accuracy is $p$ and the global order is then $p+1$ [45]. To avoid showing too many results, we only show the results for the 8th-order case, resulting in a 5th-order global design accuracy. The rate of convergence, $q_{r}$, is computed as the logarithm of the $l_{2}$ error qoutients for successively finer meshes. In Table 2 we show the rate of convergence for the conservative variables 
in the Navier-Stokes equations. In Table 3 we show the convergence rates for the conservative variables in the Euler equations.

Table 2: Convergence rates $q_{r}$ for the conservative variables in the Navier-Stokes equations

\begin{tabular}{|c|c|c|c|c|}
\hline $\mathrm{N}$ & $q_{r}(\rho)$ & $q_{r}(\rho u)$ & $q_{r}(\rho v)$ & $q_{r}(e)$ \\
\hline 64 & 4.6824 & 4.9435 & 4.8056 & 4.5442 \\
96 & 5.0336 & 4.9056 & 4.8090 & 4.5638 \\
128 & 4.8976 & 4.8315 & 4.7613 & 4.6529 \\
160 & 4.7931 & 4.7987 & 4.7459 & 4.6842 \\
192 & 4.7330 & 4.7899 & 4.7434 & 4.7013 \\
224 & 4.6970 & 4.7911 & 4.7485 & 4.7152 \\
256 & 4.6715 & 4.7972 & 4.7579 & 4.7286 \\
\hline
\end{tabular}

Table 3: Convergence rates $q_{r}$ for the conservative variables in the Euler equations

\begin{tabular}{|c|c|c|c|c|}
\hline $\mathrm{N}$ & $q_{r}(\rho)$ & $q_{r}(\rho u)$ & $q_{r}(\rho v)$ & $q_{r}(e)$ \\
\hline 64 & 4.1829 & 5.1012 & 5.1652 & 5.4700 \\
96 & 5.5570 & 5.1970 & 5.4605 & 5.4682 \\
128 & 5.7331 & 5.3679 & 5.4904 & 5.5174 \\
160 & 5.3964 & 5.3032 & 5.0674 & 5.5246 \\
192 & 5.2091 & 5.2010 & 5.0448 & 5.4999 \\
224 & 5.2630 & 5.1688 & 5.0874 & 5.4537 \\
256 & 5.3015 & 5.1357 & 5.0572 & 5.4605 \\
\hline
\end{tabular}

The functionals we consider are the volume integrals of the conservative variables and the volume integral of the pressure and the kinetic energy,

$$
\begin{array}{lll}
J_{1}(q)=\int_{\Omega} \rho d \Omega, & J_{2}(q)=\int_{\Omega} \rho u d \Omega, & J_{3}(q)=\int_{\Omega} \rho v d \Omega, \\
J_{4}(q)=\int_{\Omega} e d \Omega, & J_{5}(q)=\int_{\Omega} p d \Omega, & J_{6}(q)=\int_{\Omega} k_{e} d \Omega,
\end{array}
$$

where

$$
p=(\gamma-1)\left(e-\frac{1}{2} \rho\left(u^{2}+v^{2}\right)\right), \quad k_{e}=\frac{1}{2} \rho\left(u^{2}+v^{2}\right),
$$

are non-linear functions of the conservative variables. These functionals can be computed analytically and the rates of convergence are measured against the exact values. 
The rates of convergence for the functionals are seen in Table 4 for the Navier-Stokes equations and in Table 5 for the Euler equations.

Table 4: Convergence rates $q_{r}$ for the functionals from the Navier-Stokes equations

\begin{tabular}{|c|c|c|c|c|c|c|}
\hline $\mathrm{N}$ & $q_{r}\left(J_{1}\right)$ & $q_{r}\left(J_{2}\right)$ & $q_{r}\left(J_{3}\right)$ & $q_{r}\left(J_{4}\right)$ & $q_{r}\left(J_{5}\right)$ & $q_{r}\left(J_{6}\right)$ \\
\hline 64 & 1.0393 & 3.3961 & 2.9882 & 2.3082 & 2.3234 & 1.9452 \\
96 & 7.4099 & 7.9106 & 7.3742 & 7.3981 & 7.3492 & 8.7523 \\
128 & 7.8983 & 8.2456 & 7.0176 & 7.8020 & 7.7718 & 9.1720 \\
160 & 7.8264 & 7.9662 & 6.9992 & 7.7459 & 7.7446 & 7.8110 \\
192 & 7.7340 & 7.9598 & 7.2056 & 7.7214 & 7.7341 & 7.1184 \\
224 & 7.6973 & 8.0575 & 7.4054 & 7.7444 & 7.7622 & 6.7686 \\
256 & 7.5889 & 8.1673 & 7.4817 & 7.6570 & 7.6613 & 6.1650 \\
\hline
\end{tabular}

Table 5: Convergence rates $q_{r}$ for the functionals from the Euler equations. The last row is the constant least square (cls) fit which represent an expected value.

\begin{tabular}{|c|c|c|c|c|c|c|}
\hline $\mathrm{N}$ & $q_{r}\left(J_{1}\right)$ & $q_{r}\left(J_{2}\right)$ & $q_{r}\left(J_{3}\right)$ & $q_{r}\left(J_{4}\right)$ & $q_{r}\left(J_{5}\right)$ & $q_{r}\left(J_{6}\right)$ \\
\hline 64 & 5.1273 & 5.5316 & 8.2260 & 4.7168 & 5.1501 & 4.6192 \\
96 & 3.9332 & 8.5966 & 7.0374 & 4.1738 & 3.8252 & 8.6696 \\
128 & 8.9184 & 15.2239 & 5.3803 & 8.6687 & 9.0186 & 3.2376 \\
160 & 4.2436 & 0.1910 & 7.6280 & 5.9479 & 5.8134 & 7.1074 \\
192 & 12.4038 & 8.2731 & 12.7086 & 22.4944 & 31.5783 & 10.4366 \\
224 & 9.6461 & -2.8740 & 13.4561 & 1.5501 & -6.3680 & 6.0034 \\
256 & 8.7945 & 7.1209 & 8.2569 & 10.9129 & 2.7494 & 6.4615 \\
\hline cls & 7.5810 & 6.0090 & 8.9562 & 8.3521 & 7.3953 & 6.6479 \\
\hline
\end{tabular}

We can see from Tables 2 and 3 that the 5th-order design accuracy is nearly achieved. For the functionals we see in Table 4 that there is a clear superconvergence for the Navier-Stokes equations. These volumetric functionals are not really representative for realistic CFD applications, but they are included illustrate that the superconvergence from the linear theory derived in $[3,4]$ is present also in the nonlinear case. The convergence rates for the functionals from the Euler equations does not behave as consistently due to the lack of dissipation in the PDE itself. This could be seen also in the linear case for a smaller incompletely parabolic system similar to the Navier-Stokes equations [3]. Some artificial dissipation [26, 8] has been added, and it is possible that stronger artificial dissipation would smoothen the convergence results. Stronger dissipation would, however, increase the error constants. 


\section{Conclusions}

New far-field boundary conditions for the Navier-Stokes and Euler equations have been derived. The boundary conditions have been constructed by considering well-posedness of both the primal and dual problems. The construction ensures that the boundary conditions of the Navier-Stokes equations converge uniformly to the boundary conditions of the Euler equations, under subsonic flow conditions, as the viscosity tend to zero.

An SBP-SAT scheme were constructed which implemented the new boundary conditions. It was shown that the scheme was both energy stable and dual consistent. Stability and dual consistency implies superconvergence of any linear integral functional for linear problems. The SBP-SAT scheme were applied to the non-linear Navier-Stokes and Euler equations in conservation form and it was shown that superconvergence could be obtained also in the non-linear case with non-linear functionals.

\section{Acknowledgments}

This work has partly been carried out within the IDIHOM project which is supported by the European Commission under contract No. ACP0-GA-2010-265780.

\section{Appendix A Velocity based outflow boundary conditions}

A possible well-posed subsonic outflow boundary condition for the primal Euler equation is to set $u=0$ ( or $v=0$ ). The matrix $H_{E}$ in (18) has then to be of the form

$$
H_{E}=\left[\begin{array}{cccc}
0 & 0 & 0 & 0 \\
0 & k_{2} & 0 & 0 \\
0 & k_{3} & 0 & 0 \\
0 & k_{4} & 0 & 0
\end{array}\right]
$$

together with the other specified requirements. By following the same procedure as before, it is required that $k_{2}=k_{3}=0$. The eigenvalues of $M_{E}=A+H_{E}+H_{E}^{T}$ can then be computed as

$$
\lambda_{1,2}=\bar{u}, \quad \lambda_{3,4}=\bar{u} \pm \sqrt{\bar{c}^{2}+k_{4}^{2}+\bar{c} k_{4} \sqrt{\frac{\gamma-1}{\gamma}}}
$$


In order for $M_{E}$ to be positive semi-definite, it is required that

$$
k_{4}=-\bar{c} \sqrt{\frac{\gamma-1}{\gamma}} \pm \sqrt{\frac{\gamma\left(\bar{u}^{2}+\zeta_{4}\right)-\bar{c}^{2}}{\gamma}},
$$

where $\zeta_{4} \leq 0$ is a free parameter. The eigenvalues of $M_{E}$ are then reduced to

$$
\lambda_{1,2}=\bar{u}, \quad \lambda_{3,4}=\bar{u} \pm \sqrt{\bar{u}^{2}+\zeta_{4}} .
$$

With the above choices of $k_{2,3,4}$, the matrix $H_{E}$ is reduced to

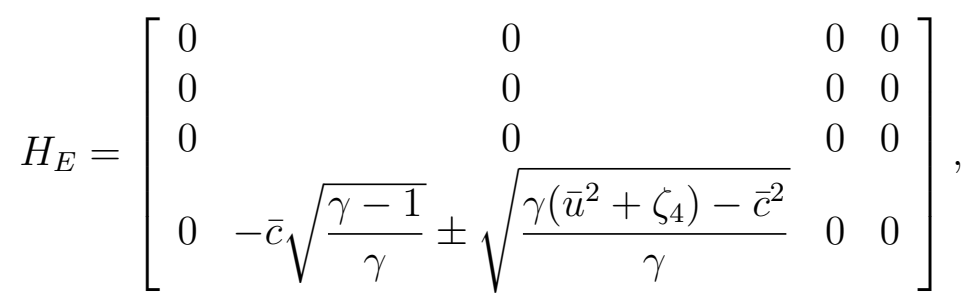

which has rank 1 as required. The free parameter will be determined such that the matrix $A+H_{E}^{T}$ has rank 3. By performing Gaussian elimination we get

$$
\begin{aligned}
& A+H_{E}^{T}=\left[\begin{array}{cccc}
\bar{u} & \bar{c} & 0 & 0 \\
\bar{c} & \bar{u} & 0 & -\sqrt{\frac{\gamma\left(\bar{u}^{2}+\zeta_{4}\right)-\bar{c}^{2}}{\gamma}} \\
\bar{\gamma} & 0 & \bar{u} & 0 \\
0 & \bar{c} \sqrt{\gamma-1} \gamma & 0 & \bar{u}
\end{array}\right] \\
& \sim\left[\begin{array}{cccc}
\bar{c} & \frac{\bar{c}}{\gamma} & 0 & 0 \\
0 & \frac{\gamma \bar{u}^{2}-\bar{c}^{2}}{\gamma \bar{u}} & 0 & -\sqrt{\frac{\gamma\left(\bar{u}^{2}+\zeta_{4}\right)-\bar{c}^{2}}{\gamma}} \\
0 & 0 & \bar{u} & 0 \\
0 & 0 & 0 & \frac{\bar{u}\left(\bar{c}^{2}-\gamma \bar{u}^{2}-\bar{c} \sqrt{\gamma-1} \sqrt{\gamma\left(\bar{u}^{2}+\zeta_{4}\right)-\bar{c}^{2}}\right)}{\bar{c}^{2}-\gamma \bar{u}^{2}}
\end{array}\right],
\end{aligned}
$$

where the last row will be zero by choosing

$$
\zeta_{4}=\frac{(\bar{c}-\bar{u})(\bar{c}+\bar{u})\left(\bar{c}^{2}-\gamma \bar{u}^{2}\right)}{(\gamma-1) \bar{c}^{2}},
$$


and hence $\operatorname{rank}\left(A+H_{E}^{T}\right)=3$. However, in order for $M_{E}$ to be positive semi-definite, it is required that $\zeta_{4} \leq 0$, which is not true for $\zeta_{4}$ in (31). For the very same reason, it is not suitable to have $\theta_{2}=0$ as the boundary condition for the dual Euler equation at the west boundary.

Remark A.1. There might be completely different choices of $k_{2,3,4}$ for which you can specify $u$ for the primal Euler at the east boundary, and $\theta_{2}$ for the dual Euler at the west boundary to obtain well-posed primal and dual problems. By following the derivational framework which worked to derive pressure based boundary conditions, however, it is not possible.

[1] S. Abarbanel and D. Gottlieb. Optimal time splitting for two- and threedimensional Navier-Stokes equations with mixed derivatives. Journal of Computational Physics, 41(1):1-33, 1981.

[2] J. Berg and J. Nordström. Stable Robin solid wall boundary conditions for the Navier-Stokes equations. Journal of Computational Physics, 230(19):7519-7532, 2011.

[3] J. Berg and J. Nordström. Superconvergent functional output for timedependent problems using finite differences on summation-by-parts form. Journal of Computational Physics, 231(20):6846-6860, 2012.

[4] J. Berg and J. Nordström. On the impact of boundary conditions on dual consistent finite difference discretizations. Journal of Computational Physics, 236(0):41-55, 2013.

[5] M. H. Carpenter, D. Gottlieb, and S. Abarbanel. Time-stable boundary conditions for finite-difference schemes solving hyperbolic systems: Methodology and application to high-order compact schemes. Journal of Computational Physics, 111(2):220-236, 1994.

[6] M. H. Carpenter, J. Nordström, and D. Gottlieb. A stable and conservative interface treatment of arbitrary spatial accuracy. Journal of Computational Physics, 148(2):341-365, 1999.

[7] M. H. Carpenter, J. Nordström, and D. Gottlieb. Revisiting and extending interface penalties for multi-domain summation-by-parts operators. Journal of Scientific Computing, 45(1-3):118-150, 2010. 
[8] P. Diener, E. N. Dorband, E. Schnetter, and M. Tiglio. Optimized high-order derivative and dissipation operators satisfying summation by parts, and applications in three-dimensional multi-block evolutions. Journal of Scientific Computing, 32(1):109-145, 2007.

[9] B. Gustafsson, H.-O. Kreiss, and J. Oliger. Time Dependent Problems and Difference Methods. Wiley Interscience, 1995.

[10] J. S. Hesthaven and D. Gottlieb. A stable penalty method for the compressible Navier-Stokes equations. I. Open boundary conditions. SIAM Journal on Scientific Computing, 17:579-612, 1994.

[11] J. E. Hicken. Output error estimation for summation-by-parts finite-difference schemes. Journal of Computational Physics, 231(9):3828-3848, 2012.

[12] J. E. Hicken and D. W. Zingg. Superconvergent functional estimates from summation-by-parts finite-difference discretizations. SIAM Journal on Scientific Computing, 33(2):893-922, 2011.

[13] J. E. Hicken and D. W. Zingg. Summation-by-parts operators and high-order quadrature. Journal of Computational and Applied Mathematics, 237(1):111$125,2013$.

[14] X. Huan, J. E. Hicken, and D. W. Zingg. Interface and boundary schemes for high-order methods. In the 39th AIAA Fluid Dynamics Conference, AIAA Paper No. 2009-3658, San Antonio, USA, June 2009.

[15] T. J. R. Hughes, L. P. Franca, and M. Mallet. A new finite element formulation for computational fluid dynamics: I. Symmetric forms of the compressible Euler and Navier-Stokes equations and the second law of thermodynamics. Computer Methods in Applied Mechanics and Engineering, 54(2):223-234, 1986.

[16] J. E. Kozdon, E. M. Dunham, and J. Nordström. Simulation of dynamic earthquake ruptures in complex geometries using high-order finite difference methods. Journal of Scientific Computing, 55(1):92-124, 2013.

[17] H.-O. Kreiss. Initial boundary value problems for hyperbolic systems. Communications on Pure and Applied Mathematics, 23(3):277-298, 1970.

[18] H.-O. Kreiss and J. Lorenz. Initial-Boundary Value Problems and the NavierStokes Equations. SIAM, 2004. 
[19] H.-O. Kreiss and G. Scherer. Finite element and finite difference methods for hyperbolic partial differential equations. In Mathematical Aspects of Finite Elements in Partial Differential Equations. Academic Press, 1974.

[20] H.-O. Kreiss and G. Scherer. On the existence of energy estimates for difference approximations for hyperbolic systems. Technical report, Uppsala University, Division of Scientific Computing, 1977.

[21] J. Lindström and J. Nordström. A stable and high-order accurate conjugate heat transfer problem. Journal of Computational Physics, 229(14):5440-5456, 2010 .

[22] K. Mattsson. Summation by parts operators for finite difference approximations of second-derivatives with variable coefficients. Journal of Scientific Computing, 51:650-682, 2012.

[23] K. Mattsson and M. H. Carpenter. Stable and accurate interpolation operators for high-order multiblock finite difference methods. SIAM Journal on Scientific Computing, 32(4):2298-2320, 2010.

[24] K. Mattsson and J. Nordström. Summation by parts operators for finite difference approximations of second derivatives. Journal of Computational Physics, 199(2):503-540, 2004.

[25] K. Mattsson, M. Svärd, M. Carpenter, and J. Nordström. High-order accurate computations for unsteady aerodynamics. Computers and Fluids, 36(3):636-649, 2007.

[26] K. Mattsson, M. Svärd, and J. Nordström. Stable and accurate artificial dissipation. Journal of Scientific Computing, 21(1):57-79, 2004.

[27] K. Mattsson, M. Svärd, and M. Shoeybi. Stable and accurate schemes for the compressible Navier-Stokes equations. Journal of Computational Physics, 227:2293-2316, 2008.

[28] A. Nissen, G. Kreiss, and M. Gerritsen. Stability at nonconforming grid interfaces for a high order discretization of the Schrödinger equation. Technical Report 2011-017, Uppsala University, Division of Scientific Computing, 2011.

[29] J. Nordström. The influence of open boundary conditions on the convergence to steady state for the Navier-Stokes equations. Journal of Computational Physics, 85(1):210-244, 1989. 
[30] J. Nordström. The use of characteristic boundary conditions for the NavierStokes equations. Computers \&f Fluids, 24(5):609-623, 1995.

[31] J. Nordström and M. H. Carpenter. Boundary and interface conditions for highorder finite-difference methods applied to the Euler and Navier-Stokes equations. Journal of Computational Physics, 148(2):621-645, 1999.

[32] J. Nordström and M. H. Carpenter. High-order finite difference methods, multidimensional linear problems, and curvilinear coordinates. Journal of Computational Physics, 173(1):149-174, 2001.

[33] J. Nordström, S. Eriksson, C. Law, and J. Gong. Shock and vortex calculations using a very high order accurate Euler and Navier-Stokes solver. Journal of Mechanics and MEMS, 1(1):19-26, 2009.

[34] J. Nordström, J. Gong, E. van der Weide, and M. Svärd. A stable and conservative high order multi-block method for the compressible Navier-Stokes equations. Journal of Computational Physics, 228(24):9020-9035, 2009.

[35] J. Nordström and B. Lönn. Energy decay of vortices in viscous fluids: An applied mathematics view. Journal of Fluid Mechanics, 709:593-609, 2012.

[36] J. Nordström and M. Svärd. Well-posed boundary conditions for the NavierStokes equations. SIAM Journal on Numerical Analysis, 43(3):1231-1255, 2005.

[37] T. H. Pulliam. The Euler equations, 1994. NASA Ames Research Center.

[38] L. Shunn, F. Ham, and P. Moin. Verification of variable-density flow solvers using manufactured solutions. Journal of Computational Physics, 231(9):38013827, 2012.

[39] B. Strand. Summation by parts for finite difference approximations for $\mathrm{d} / \mathrm{dx}$. Journal of Computational Physics, 110(1):47-67, 1994.

[40] G. Strang. Accurate partial difference methods. Numerische Mathematik, 6(1):37-46, 1964.

[41] J. C. Strikwerda. Initial boundary value problems for incompletely parabolic systems. Communications on Pure and Applied Mathematics, 30(6):797-822, 1977.

[42] M. Svärd. On coordinate transformations for summation-by-parts operators. Journal of Scientific Computing, 20:29-42, 2004. 
[43] M. Svärd, M. H. Carpenter, and J. Nordström. A stable high-order finite difference scheme for the compressible Navier-Stokes equations, far-field boundary conditions. Journal of Computational Physics, 225(1):1020-1038, 2007.

[44] M. Svärd, K. Mattsson, and J. Nordström. Steady-state computations using summation-by-parts operators. Journal of Scientific Computing, 24(1):79-95, 2005.

[45] M. Svärd and J. Nordström. On the order of accuracy for difference approximations of initial-boundary value problems. Journal of Computational Physics, 218(1):333-352, 2006. 